\title{
Iron $\mathrm{K} \alpha$ Spectra from an Atomic Modeling Perspective
}

\author{
Duane A. Liedahl \\ Department of Physics and Advanced Technologies, Lawrence Livermore \\ National Laboratory, P.O. Box 808, L-473, Livermore, CA 94550
}

\begin{abstract}
In order to upgrade the diagnostic capability associated with $\mathrm{Fe}$ $\mathrm{K} \alpha$ spectra, we have calculated detailed atomic models that include the effects of inner-shell photoionization, autoionization, collisional excitation at relatively high particle number densities, and photoexcitation in plasmas with high X-ray and/or UV energy densities. I discuss some of the potential uses of these models in the context of compact $\mathrm{X}$-ray sources.
\end{abstract}

\section{Introduction}

Observations and analysis of $\mathrm{Fe} \mathrm{K} \alpha$ spectra have long been a mainstay of astrophysical X-ray spectroscopy. This will continue to be true in the future, with Astro-E 2 and Constellation- $X$ in the offing. Solar X-ray observations (Seely, Feldman, \& Safranova 1986) and laboratory plasma experiments (Beiersdorfer et al. 1993) have shown that Fe K $\alpha$ spectra can be extremely complex, comprising several charge states crowding a relatively narrow spectral band. This is not observed in extra-solar objects, since, to date, the data have been of poor spectral resolution. Advancements in detector capabilities, however, exemplified by the XRS on Astro-E 2, promise to uncover the spectral complexity in the $\mathrm{Fe}$ $\mathrm{K} \alpha$ spectrum in several classes of celestial X-ray source.

Owing to the poor resolving power with which Fe K spectra are measured, analyzes are typically restricted to dealing with "the iron line," even if the underlying structure is suspected to be rich; obviously, it would be ill-advised to apply a complicated spectral model to a feature that can be described with a simple Gaussian model. Unfortunately, many potentially useful plasma diagnostics become obscured by detectors with poor resolution, which, in turn, tends to inhibit the development of these diagnostics. In light of the imminent launch of Astro-E 2, with a resolving power $\approx 6 \mathrm{eV}$, this situation is improving, and detailed calculations of the atomic physics processes associated with Fe $\mathrm{K}$ spectral formation, using state-of-the-art computer codes, are now becoming available (for example, Palmeri et al. 2003).

We have used the HULLAC (Hebrew University/Lawrence Livermore Atomic Code; Bar-Shalom et al. 1988) atomic physics package to search for plasma diagnostics involving Fe $\mathrm{K}$ spectra. Thus far, this research has focused on the L-shell species (Fe XVII-XXIV), which is a natural follow-on to similar work on Si K $\alpha$ spectra, which was motivated by an observation of the high-mass X-ray binary Vela X-1 using the high-resolution gratings on the Chandra satellite (Liedahl et al., in prep.). We find that the part of the Si $\mathrm{K} \alpha$ line complex formerly attributed to neutral or near-neutral species (Sako et al. 1999) is actually made 
up of features corresponding to Si III - Si XII. The K $\alpha$ spectra of L-shell ions provide unique diagnostic capability, and provide information on plasmas that exist at an intermediate range of ionization parameter. There is the possibility that, at least in some sources, the $\mathrm{Fe} \mathrm{K} \alpha$ complex will be observed as consisting of several charge states, some of which are L-shell ions. Not only will such detections provide corollary or corroborative information relative to $\mathrm{K} \alpha$ spectra from lower- $Z$ elements, but $\mathrm{Fe} \mathrm{K} \alpha$ spectra, typically possessing larger equivalent widths than lower- $Z$ elements, might be found to contain the superior set of diagnostics.

\section{Results}

The main results of this research are described below. A more thorough presentation will appear in a future paper.

\subsection{Density Diagnostics}

A detailed treatment of the level population kinetics shows that the $\mathrm{K} \alpha$ fluorescence spectrum can be a proxy for the local electron density. Underlying the mechanism is collisional excitation from ground to low-lying meta-stable levels, which, when photoionized out of the 1s shell, leads to fluorescence lines that are distinct from those produced in the low-density limit (Jacobs et al. 1989). The critical electron densities are $\sim 10^{13} \mathrm{~cm}^{-3}$ (Liedahl et al. 1992).

\subsection{Direct Photoexcitation by a Continuum Radiation Field}

Sources characterized by a hard X-ray continuum, in which fluorescence following inner-shell photoionization can be important, can also host gas for which direct photoexcitation of bound levels can drive line emission. This has been shown for the case of Seyfert 2 galaxies (Kinkhabwala et al. 2002) and high-mass Xray binaries (Wojdowski et al. 2003). Similarly, line emission from quasi-bound auto-ionizing levels can be driven by the continuum radiation field. The essential difference, as found in this work, is that photoexcitation of auto-ionizing levels can give rise to bright lines that are not strongly driven by any other process under conditions of photoionization equilibrium. Lines of this type thus provide relatively unambiguous constraints on the column densities of the relevant charge states.

\subsection{Level Population Dependence of Resonant Auger Destruction}

Resonant Auger destruction (Ross, Fabian, \& Brandt 1996) can efficiently suppress $\mathrm{K} \alpha$ emission from $\mathrm{L}$-shell ions, since, as opposed to simple resonant scattering by bound levels, when the upper level is autoionizing, the photon destruction probability per scatter is equal to the autoionization branching ratio, which often exceeds 0.5. The other quantities of interest are, of course, the line optical depths. For a given fluorescence line, the optical depth depends on the level population density of the lower level of the radiative transition, which, in turn, depends on the local electron density. Therefore, the efficacy of resonant Auger destruction depends on both the ionic column density, a macroscopic quantity, and the local electron density, a microscopic quantity. It has been found that 
there are bright $\mathrm{K} \alpha$ fluorescence lines from Fe L-shell ions that may survive the resonant Auger destruction process and escape to infinity. To compare with the case of Vela $\mathrm{X}-1$, as mentioned above, where we clearly see $\mathrm{K} \alpha$ lines from $\mathrm{Si}$ L-shell ions, the fluorescing gas in that object appears to be of relatively low ionic column density. We have found a set of transitions that can survive large ionic column densities, such as may be found in accretion disk atmospheres, for example. The action of resonant Auger destruction can produce spectra that differ dramatically from the "zero-D" limit.

\section{Concluding Remarks}

Through detailed treatments of atomic level population kinetics, we have found that $\mathrm{Fe} \mathrm{K} \alpha$ spectra are, at least on "paper," exceptionally versatile diagnostics of physical conditions in X-ray photoionized plasmas, such as are found in active galactic nuclei and X-ray binaries. Since the spectra can differ dramatically from what one might expect from an assessment of a "zero-D," isolated ion model, care must be taken in identifying individual fluorescence lines. This will clear the way for the application of the rich diagnostic potential of these ions.

Acknowledgments. D.A.L. was supported in part by the NASA Long Term Space Astrophysics Program grant S-92654-F. Work at LLNL was performed under the auspices of the U.S. Department of Energy by the University of California Lawrence Livermore National Laboratory under contract No. W7405-Eng-48.

\section{References}

Bar-Shalom, A., Klapisch, M., \& Oreg, J. 1988, Phys.Rev.A, 38, 1773

Beiersdorfer, P., Phillips, T., Jacobs, V.L., Hill, K.W., Bitter, M., \& von Goeler, S. 1993, ApJ, 409, 846

Jacobs, V.L., Doschek, G.A., Seely, J.F., \& Cowan, R.D. 1989, Phys.Rev.A, 39, 2411

Kinkhabwala, A., et al. 2002, ApJ, 575, 732

Liedahl, D.A., Kahn, S.M., Osterheld, A.L., \& Goldstein, W.H. 1992, ApJ, 391, 306

Palmeri, P., Mendoza, C., Kallman, T.R., \& Bautista, M.A. 2003, A\&A, 403, 1175

Ross, R.R., Fabian, A.C, \& Brandt, W.N. 1996, MNRAS, 278, 1082

Sako, M., Liedahl, D.A., Kahn, S.M., \& Paerels, F. 1999, ApJ, 525, 921

Seely, J.F., Feldman, U., \& Safranova, U.I. 1986, ApJ, 304, 838

Wojdowski, P.S., Liedahl, D.A., Sako, M., Kahn, S.M., \& Paerels, F. 2003, ApJ, 582, 959 